\title{
Extent of transmission captured by contact tracing in a tuberculosis high endemic setting
}

\author{
To the Editor:
}

We have previously documented a high incidence of tuberculosis (TB) (>1000 per 100000 inhabitants) and considerable Mycobacterium tuberculosis transmission in East Greenland despite population-wide screenings implemented by the Greenland health authorities [1-5]. In Greenland, all contacts of TB patients are routinely screened for $M$. tuberculosis infection and TB. Individuals with newly acquired M. tuberculosis infection are offered 6 months of preventive isoniazid treatment. Despite the considerable resources spent on clinical examinations and preventive treatment over the years, TB incidence rates have remained high in East Greenland [1].

In a recent study using whole genome sequencing (WGS), we found culture-positive TB cases in East Greenland to be caused by genetically nearly identical M. tuberculosis strains [4]. However, for many of the TB cases, results of the WGS suggested direct $M$. tuberculosis transmission from source cases not identified by contact-tracing efforts.

To further explore this, we aimed to estimate the percentage of M. tuberculosis culture-positive TB cases with or without a reported contact confirmed by WGS as a potential source, thereby providing evidence of M. tuberculosis transmission occurring outside traditional contact tracing circles.

East Greenland is an isolated part of Greenland, inhabited by only 6\% $(n=3514)$ of the total Greenland population [6]. Inhabitants live in two towns and five smaller settlements. At birth, all Greenland citizens receive a personal identifier from the civil registration system, which enables a unique linkage of personal data across public registries.

In Greenland, TB is mandatorily notifiable and contact tracing is routinely performed for all TB cases. Information on all notified TB cases from East Greenland $(n=134)$ in the period from 2008 to 2012 and their reported contacts were sought from the TB notification system. Detailed contact tracing data were available for $80 \%(n=107)$ of notified cases, amounting to a total of 1241 designated contacts and numbering an average of 12 contacts per TB case. The Committee for Scientific Research in Greenland approved the study (approval no. 2012-071304). The project was reported to and followed all instructions from the Danish Data Protection Agency.

As previously reported [4], isolates from 182 culture-positive cases from 1992 to 2012 (98\% of all culture-positive cases) have already been analysed by WGS. In short, M. tuberculosis isolates were retrieved from the nationwide strain collection at Statens Serum Institut. After DNA extraction [1], libraries were prepared with Nextera XT kits and run on Illumina next-generation sequencing platforms (Illumina, San Diego, CA, USA). An overall maximum parsimony tree including the 182 culture-positive TB cases was created with Bionumerics, version 7.5 (Applied Math, Sint-Martens-Latem, Belgium) based on 1385 concatenated single nucleotide polymorphisms (SNPs) [4]. For further details on variant calling, reference mapping and SNP filters, please refer to our previously published paper that also describes how raw data in the form of fastq files were submitted to the EMBL-EBI ENA sequence read archive [4]. In total, 287 TB cases were notified from 1992 to 2012. Of the remaining 105 cases not included in the previous study, 91 had at least one diagnostic sample registered, of which 84 were culture- and microscopy-negative [4].

@ERSpublications

In a TB endemic setting $>50 \%$ of culture-positive TB cases were likely infected via community acquired transmission http://ow.ly/Rqjd307cf6o

Cite this article as: Bjorn-Mortensen $\mathrm{K}$, Lillebaek $\mathrm{T}$, Koch $\mathrm{A}$, et al. Extent of transmission captured by contact tracing in a tuberculosis high endemic setting. Eur Respir J 2017; 49: 1601851 [https://doi.org/ 10.1183/13993003.01851-2016]. 
The previously conducted WGS study [4] enabled us to compare contract-tracing data obtained from 2008 to 2012 with the overall phylogenetic tree representing cases diagnosed from 1992 to 2012. We evaluated whether isolates linked by contact-tracing data were within a genetic distance suggestive of likely direct transmission. To display this relationship, we compared links identified by contact tracing with WGS-suggested links. We used a maximum distance of five SNPs between isolates as threshold for likely direct transmission, as previously suggested by other authors [7]. We recently showed that M. tuberculosis transmission in this setting most likely occurs with strains within an even smaller genetic distance [4], and considered this to be a conservative measure of direct transmission. Sequencing and contact tracing data were compared with NodeXL ${ }^{\oplus}$ (Socialmedia Research Foundation).

Of the 134 TB cases notified from 2008 to 2012, 86 (64\%) were culture-positive and therefore included in the WGS analyses. For the remaining culture-negative TB cases, diagnosis was made by clinical criteria only $(\mathrm{n}=47)$ and for one culture-negative case by positive microscopy. Of the 86 culture-positive TB cases, 84 had available contact-tracing data, of which $52(60 \%)$ were reported as a contact of another culture-positive case. Of the 86 culture-positive cases included in the WGS analyses, 79 were classified as pulmonary cases. Of the remaining $48 \mathrm{~TB}$ cases, 41 were pulmonary TB cases.

However, when contact-tracing data were challenged with the genetic relationship of included isolates, only $40(47 \%)$ of the 86 culture-positive TB cases were within a maximum distance of five SNPs from an isolate from any reported contact. Thus, a likely source of infection among reported contacts was only identified for $47 \%$. Thus in this isolated high TB incidence setting, 53\% of the culture-positive TB cases had no reported contact(s) within a genetic distance suggestive of direct transmission (figure 1), despite including all available contact-tracing data and with no regard to smear positivity or the direction of the reported link. Thus, for these cases, traditional contact tracing did not reveal the source of infection despite an average of 12 screened contacts per case.

Our finding from a unique, isolated, high TB incidence setting without HIV or multidrug-resistant TB confirms previous reports using epidemiological data and/or other subtyping methods, which show that community-, rather than reported contact-acquired, transmission is likely in high TB incidence areas [8-12]. For example, a recent study from Malawi using WGS reported a very similar percentage of less than $50 \%$ of culture-positive cases; this was explained by transmission from a close contact [13]. Our study is important in order to demonstrate that in high-incidence areas, sources of TB transmission should not only be sought among close contacts.

In areas with a high $M$. tuberculosis infection pressure, contact tracing is challenged by the large number of potential community contacts not included in the screening efforts. Thus, in an area like East Greenland and similar settings worldwide, it seems relevant to include alternative methods to identify TB cases infected through community-acquired transmission. In East Greenland, teenagers, BCG-unvaccinated individuals and inhabitants of previously low endemic areas are particular risk groups $[1,2]$. However, the TB incidence in East Greenland is considerably higher than in the rest of Greenland (1000 versus 150 per
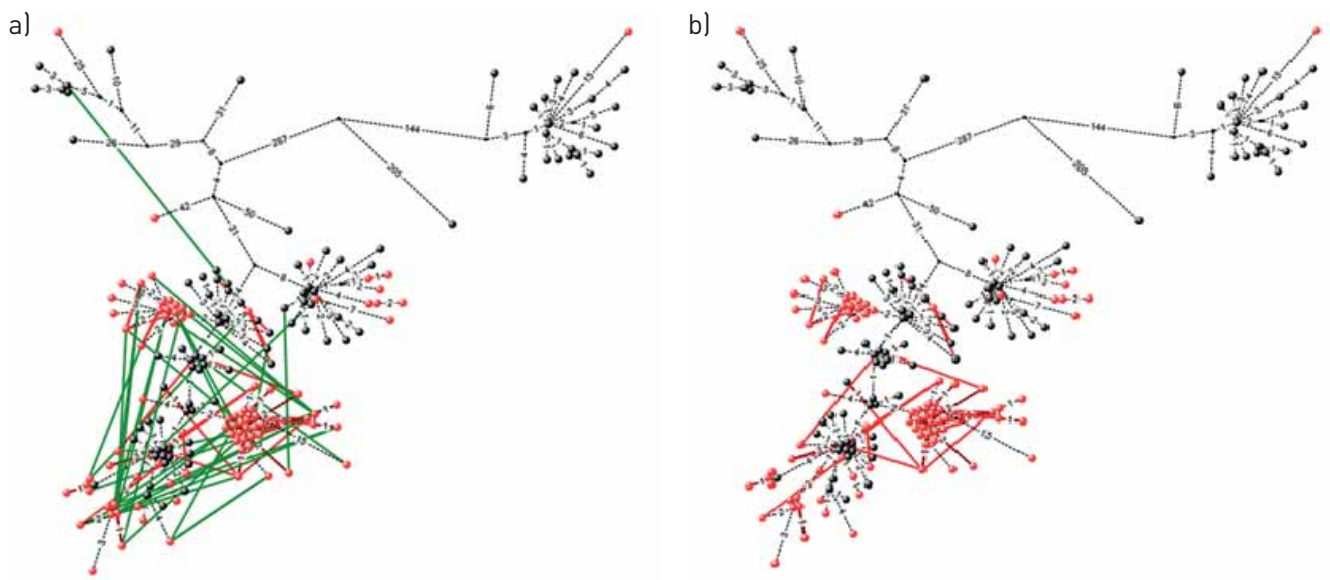

FIGURE 1 An overall maximum parsimony tree based on 1385 concatenated single nucleotide polymorphisms (SNPs) showing the genomic links (black) between 182 culture-positive tuberculosis (TB) cases from East Greenland, 1992 to 2012, compared with contact-tracing links. Only contact-tracing links from culture-positive TB cases from 2008 to 2012 (red) are included in the analyses. a) Any identified link ( $n=70$, green and red) between included TB cases $(n=52)$. b) Only contact tracing links $(n=40)$ between cases $(n=40)$ with a maximum distance of five SNPs (red), suggestive of direct transmission between cases. 
100000 inhabitants) and perhaps the entire East Greenland population should be considered at risk. According to World Health Organization guidelines, systematic screenings for active TB of geographically defined subpopulations with a high prevalence of undetected TB are sometimes needed [14].

The major strengths of this study were the completeness of included samples, the isolated geographical setting and the unique data linkage in combination with WGS at a population-based level. The study has two major limitations; contact-tracing data were missing for $20 \%(n=27)$ of the 134 cases notified 2008 to 2012 and only culture-positive cases $(n=86)$ were available for WGS analyses. While missing contact-tracing data could lead to underestimation of the true number of possible contacts, only two of the cases with missing data were culture-positive and only one was a smear microscopy-positive pulmonary case. The remainder were therefore less likely to be sources of $M$. tuberculosis transmission and thus we do not believe that our result would have changed greatly if they had been included. In contrast, including all culture-positive cases with no regard to smear positivity could potentially lead to overestimation of the number of possible contacts.

In conclusion, 53\% of culture-positive TB cases in East Greenland were most likely infected by a source not identified through traditional contact tracing. Thus, this study illustrates the difficulties in confining M. tuberculosis transmission through contact tracing alone in high TB incidence settings. This result has potentially important implications for future TB control measures in TB high endemic regions, as it leads to considerations on further actions needed for TB control and how best to use the resources available; e.g. in small, high $\mathrm{TB}$ incidence communities, population-wide $\mathrm{TB}$ screenings could be considered as an alternative to traditional contact tracing.

Karen Bjorn-Mortensen ${ }^{1,2,3,4}$, Troels Lillebaek ${ }^{2}$, Anders Koch $^{1}$, Bolette Soborg ${ }^{1}$, Karin Ladefoged $^{5}$, Hans Christian Florian Sørensen ${ }^{6}$, Thomas Andreas Kohl ${ }^{7,8}$, Stefan Niemann ${ }^{7,8}$ and Aase Bengaard Andersen ${ }^{4,9}$

${ }^{1}$ Dept of Epidemiology Research, Statens Serum Institut, Copenhagen, Denmark. ${ }^{2}$ International Reference Laboratory of Mycobacteriology, Statens Serum Institut, Copenhagen, Denmark. ${ }^{3}$ Greenland's Center of Health Research, Nuuk, Greenland. ${ }^{4}$ Research Unit for Infectious Diseases, Institute of Clinical Medicine, University of Southern Denmark, Odense, Denmark. ${ }^{5}$ Dept of Internal Medicine, Queen Ingrid's Hospital, Nuuk, Greenland. ${ }^{6}$ Tasiilaq Hospital, Tasiilaq, Greenland. ${ }^{7}$ Molecular and Experimental Mycobacteriology, Forschungszentrum Borstel, Leibniz-Zentrum für Medizin und Biowissenschaften, Borstel, Germany. ${ }^{8}$ German Center for Infection Research (DZIF), partner site Borstel, Borstel, Germany. ${ }^{9}$ Dept of Infectious Diseases, Copenhagen University Hospital, Copenhagen, Denmark.

Correspondence: Karen Bjorn-Mortensen, Dept of Epidemiology Research/International Reference Laboratory of Mycobacteriology, Artillerivej 5, 2300 Copenhagen, Denmark. E-mail: kabm@ssi.dk

Received: Sept 202016 | Accepted after revision: Nov 232016

Support statement: The Commission for Scientific Research in Greenland, Forskningspuljen and Sundhedspuljen (Greenlandic Self-Government), (0605-00062B) funded the study. Parts of the work were supported by the Seed Funding Initiative for Collaboration of the Universities of Southern Denmark, Kiel, Hamburg and Aarhus, Lundbeck Foundation (R151-2013-14628), Novo Nordisk Foundation (7651), the European Union TB-PAN-NET (FP7-223681), PathoNgenTrace (FP7-278864-2) projects, and the German Center for Infection Research (DZIF). The funders had no role in study design, data collection and analysis, decision to publish, or preparation of the manuscript. Funding information for this article has been deposited with the Open Funder Registry.

Conflict of interest: Disclosures can be found alongside this article at erj.ersjournals.com

Acknowledgements: The authors thank the hospital staff in Tasiilaq for identifying and treating TB patients, the National Board of Health for support and access to data, and laboratory staff at the IRLM and Forschungszentrum Borstel for DNA extraction, library preparation and WGS. All authors contributed to the conception and design of the study, the analyses of data and writing of the article.

\section{References}

1 Bjorn-Mortensen $\mathrm{K}$, Andersen AB, Koch A, et al. Tuberculosis outbreak in East Greenland: groups at risk in an isolated arctic setting. Eur Respir J 2015; 46: 865-869.

2 Michelsen SW, Soborg B, Koch A, et al. The effectiveness of BCG vaccination in preventing Mycobacterium tuberculosis infection and disease in Greenland. Thorax 2014; 69: 851-856.

3 Soborg B, Koch A, Thomsen VØ, et al. Ongoing tuberculosis transmission to children in Greenland. Eur Respir J 2010; 36: 878-884.

4 Bjorn-Mortensen K, Soborg B, Koch A, et al. Tracing Mycobacterium tuberculosis transmission by whole genome sequencing in a high incidence setting: a retrospective population-based study in East Greenland. Sci Rep 2016; 6: 33180.

5 Naalakkersuisut, Government of Greenland. National TB strategi 2012-2016 [National TB Strategy 2012-2016]. http://dk.vintage.nanoq.gl/Service/Publikationer/Udgivelser/2011/Tuberkulose_strategi.aspx Date last accessed: January $17,2017$.

6 Grønlands Statistik. www.stat.gl Date last accessed: April 8, 2012.

7 Walker TM, Ip CLC, Harrell RH, et al. Whole-genome sequencing to delineate Mycobacterium tuberculosis outbreaks: a retrospective observational study. Lancet Infect Dis 2013; 13: 137-146.

8 Middelkoop K, Bekker L-G, Morrow C, et al. Decreasing household contribution to TB transmission with age: a retrospective geographic analysis of young people in a South African township. BMC Infect Dis 2014; 14: 221. 
9 Chamie G, Wandera B, Marquez C, et al. Identifying locations of recent TB transmission in rural Uganda: a multidisciplinary approach. Trop Med Int Health 2015; 20: 537-545.

10 Verver S, Warren RM, Munch Z, et al. Proportion of tuberculosis transmission that takes place in households in a high-incidence area. Lancet 2004; 363: 212-214.

11 Classen CN, Warren R, Richardson M, et al. Impact of social interactions in the community on the transmission of tuberculosis in a high incidence area. Thorax 1999; 54: 136-140.

12 Sia IG, Buckwalter SP, Doerr KA, et al. Genotypic characteristics of Mycobacterium tuberculosis isolated from household contacts of tuberculosis patients in the Philippines. BMC Infect Dis 2013; 13: 571.

13 Glynn JR, Guerra-Assunção JA, Houben RMGJ, et al. Whole Genome Sequencing Shows a Low Proportion of Tuberculosis Disease Is Attributable to Known Close Contacts in Rural Malawi. PloS One 2015; 10: e0132840.

14 World Health Organization. Systematic Screening for Active Tuberculosis: Principles and Recommendations. Geneva, World Health Organization, 2013. Available from: http://apps.who.int/iris/bitstream/10665/84971/1/ 9789241548601_eng.pdf?ua=1 